\title{
Erratum
}

\section{Quasi-static mode III fracture in a nonhomogeneous viscoelastic body}

Acta Mechanica 85, $235-249(1990)$

J. M. Herrmann, College Station, and L. Schovanec, Lubbock, Texas

If $\alpha$ is negative, the transfer function $T(p),(2.13)$, has a zero of order $p^{2}$ at $p=0$. This necessitates a different factorization of $T(p)$ than in the case of $\alpha$ positive so that $\log (T(p))$ is well defined. The correct factorization of this case is $T(p)=T_{1}(p) T_{2}(p) T_{3}(p)$ where

$$
\begin{aligned}
& T_{1}(p)=-\tilde{\mu}(i v p), \quad T_{2}(p)=\frac{2 p^{2}}{\sqrt{\alpha^{2}+4 p^{2}}}, \\
& T_{3}(p)=\left(\alpha+\sqrt{\alpha^{2}+4 p^{2}}\right) \frac{\sqrt{\alpha^{2}+4 p^{2}}}{4 p^{2}}=1+\frac{\alpha^{2}}{4 p^{2}}\left[1-\sqrt{1+\frac{4 p^{2}}{\alpha^{2}}}\right] .
\end{aligned}
$$

Note that $1 / 2 \leqq T_{3}(p) \leqq 1$ for all real $p$.

The homogeneous solutions $X_{i}^{+}(p)=T_{i}(p) X_{i}^{-}(p)$ are then given by

$$
\begin{aligned}
& X_{1}{ }^{+}(z)=1, \quad X_{1}{ }^{-}(z)=\frac{-1}{\tilde{\mu}(i v z)}, \\
& X_{2}{ }^{+}(z)=\frac{z}{\omega^{+}(z+i|\alpha / 2|)}, \quad X_{2}{ }^{-}(z)=\frac{\omega^{-}(z-i|\alpha / 2|)}{z},
\end{aligned}
$$

and

$X_{3}{ }^{ \pm}(z)=\exp \left(\Gamma^{ \pm}(z)\right) \quad$ where $\quad \Gamma^{ \pm}(z)=\frac{1}{2 \pi i} \int_{-\infty}^{\infty} \frac{\log \left(T_{3}(\tau)\right)}{\tau-z} d \tau$

where

$T_{3}(p)=1+\frac{\alpha^{2}}{4 p^{2}}\left[1-\frac{2}{|\alpha|} \omega^{+}(p+i|\alpha / 2|) \omega^{-}(p-i|\alpha / 2|)\right]$

is analytic in the lower half plane except along the branch cut $B=\{z=-i q$ where $|\alpha / 2| \leqq q<\infty\}$. Furthermore, it can be shown that $\operatorname{Re}\left[T_{3}(z)\right]>0$ for all $z$ in the lower half plane except at $z=-i \alpha / 2$ where $\operatorname{Re}\left[T_{3}(-i \alpha / 2)\right]=0$. Thus an analytic branch of $\log \left(T_{3}(z)\right)$ can be formed for $z$ in the lower half plane except along the branch cut $B$. Then $\Gamma^{ \pm}(z)$ can be simplified by residues in a similar manner as $\alpha$ positive and the solution to the homogeneous $\mathrm{R}-\mathrm{H}$ problem for $\alpha$ negative is determined to be

$$
X^{+}(z)=\frac{z \exp (I(z))}{\omega^{+}(z+i|\alpha / 2|)}, \quad X^{-}(z)=\frac{-1}{\tilde{\mu}(i v z)} \frac{\omega^{-}(z-i|\alpha / 2|)}{z} \frac{\exp (I(z))}{T_{3}(z)} \quad \text { for } z \notin B,
$$


$X_{3}{ }^{-}(-i q)=\frac{-e^{i \pi / 4}}{\tilde{\mu}(v q)} \frac{\sqrt{q+|\alpha / 2|}}{q} \frac{\exp (I(-i q))}{\sqrt{1-\alpha^{2} /\left(4 q^{2}\right)}}$ for $|\alpha / 2|<q<\infty$

where

$I(z)=\frac{-1}{\pi} \int_{1}^{\infty} \arctan \left(\frac{1}{\sqrt{u^{2}-1}}\right) \frac{d u}{u-|2 / \alpha| i z} \quad$ for $z \notin B$

and

$I(-i q)=P V \frac{-1}{\pi} \int_{1}^{\infty} \arctan \left(\frac{1}{\sqrt{u^{2}-1}}\right) \frac{d u}{u-|2 / \alpha| q} \quad$ for $|\alpha / 2|<q<\infty$.

From the expression for the ERR (3.9) and the homogeneous solutions above, it is found that the ERR has the nondimensional form for $\alpha$ negative of

$G=\frac{L_{e}^{2} a_{e}}{2 \mu_{\infty}} \frac{1-\varepsilon}{1+\varepsilon} \frac{1}{\tilde{m}(\gamma / \varepsilon)}(1+x / 2) h(x, \varepsilon) e^{-2 I_{1}+I_{2}+I_{3}}$

where

$h(\varkappa, \varepsilon)=\frac{1}{\sqrt{1-(\varkappa \varepsilon / 2)^{2}}} \quad$ for $\quad x \varepsilon<2$,

$h(x, \varepsilon)=\frac{1}{\sqrt{(\varkappa \varepsilon / 2)^{2}-1}\left((x \varepsilon / 2)+\sqrt{(x \varepsilon / 2)^{2}-1}\right)} \quad$ for $\quad x \varepsilon>2$,

with $I_{1}, I_{2}$, and $I_{3}$ as in (3.12).

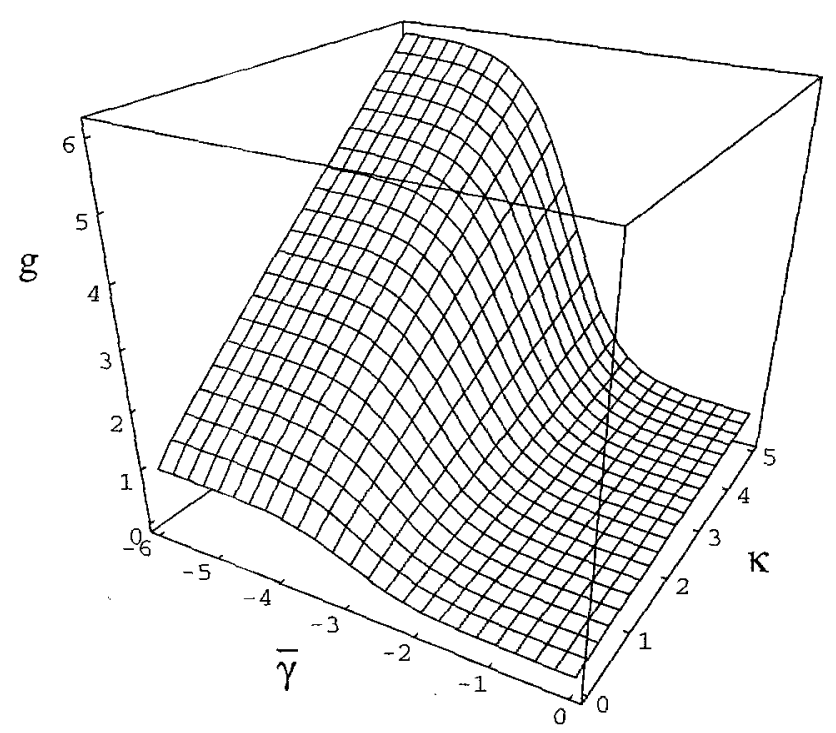

Fig. 1. The normalized ERR $g$ for $\alpha<0$ versus the inhomogeneity parameter $\varkappa=|\alpha| a_{\mathrm{e}}$ and $\bar{\gamma}=\log (\gamma)$ $=\log \left(\tau v / a_{e}\right)$ 


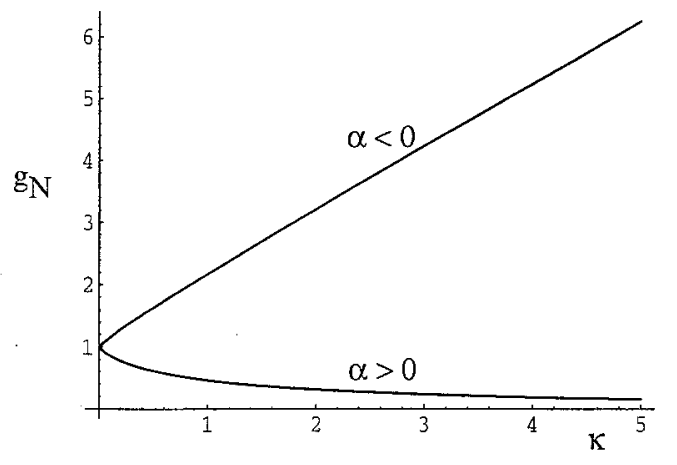

Fig. 2. The normalized ERR with no failure zone versus the inhomogeneity parameter $\varkappa=|\alpha| a_{e}$

The ERR if $\alpha$ is negative for the special cases of no failure zone and an elastic material are

$G_{n f}=\frac{L_{e}^{2} a_{e}}{2 \mu_{\infty}} \frac{1}{m(0)}(1+x / 2) e^{-2 I_{1}}=\frac{K_{e}{ }^{2}}{2 \mu(0)}$

$G_{e}=\frac{L_{e}{ }^{2} a_{e}}{2 \mu} \frac{1-\varepsilon}{1+\varepsilon}(1+x / 2) h(\varkappa, \varepsilon) e^{-2 I_{1}+I_{2}+I_{3}}$.

The asymptotic expansion for the ERR as $x \rightarrow 0$ for $\alpha$ negative has the form

$G=G_{h}\left[1+\frac{-1}{\pi} x \ln (x)+k_{0} x+o(x)\right] \quad$ as $\quad k \rightarrow 0$

where $k_{0}=\frac{1}{2}+\frac{1}{\pi}(\ln (2)+c)=.75958$. Thus it can be seen that for small values of $\varkappa$ the effect of material inhomogeneity is to increase the ERR for a material which is more rigid near the plane of the crack.

The corrected Figs. 3 and 4 are given above (Figs. 1 and 2 here).

Author's addresses: Dr. J. M. Herrmann, Department of Mathematics, Texas A \& M University, College Station, TX 77843, and Dr. L. Schovanec, Department of Mathematics, Texas Tech University, Lubbock, TX 79409, U.S.A.

Verleger: Springer-Verlag KG, Sachsenplatz 4-6, A-1201 Wien. - Herausgeber: Prof. Dipl.-Ing. Dr. Hans Troger und Prof Dipl.-Ing. Dr. Franz Ziegler, beide Technische Universität, Karlsplatz 13, A-1040 Wien. - Redaktion: Karlsplatz 13 A-1040 Wien. - Hersteller: Druckerei zu Altenburg GmbH, D-04600 Altenburg - Verlagsort: Wien. - Herstellungsort:
Altenburg. - Printed in Germany. 